DF/IST-6.97

October 1997

\title{
Primordial Density Fluctuations in a Dual Supergravity Cosmology
}

\author{
M. C. Bento, O. Bertolami and N. J. Nunes \\ Departamento de Física, Instituto Superior Técnico \\ Av. Rovisco Pais, 1096 Lisboa Codex, Portugal
}

\begin{abstract}
We analyse the spectrum of energy density fluctuations of a dual supergravity model where the dilaton and the moduli are stabilized and sucessful inflation is achieved inside domain walls that separate different vacua of the theory. Constraints on the parameters of the superpotential are derived from the amplitude of the primordial energy density fluctuations as inferred from $\mathrm{COBE}$ and it is shown that the scale dependence of the tensor perturbations nearly vanishes.
\end{abstract}


Measurements of the cosmic microwave background anisotropy can be used to test in detail the inflationary hypothesis within the next decade. Hence, it is important to study field-theoretic inflation in a realistic context. It is thought that physics below the Planck scale is described by a unified theory of all the fundamental interactions incorporating supersymmetry. The local version of supersymmetry - supergravity - includes gravity and is, therefore, particularly relevant to build inflationary models in the supergravity context. A generic problem for a wide variety of supergravity models is that the effective potential for the would-be inflaton field, $\phi$, is too steep, growing as $\exp \left(\frac{\phi^{2}}{M^{2}}\right)$ for large $\phi$, to allow a sufficiently long period of inflation to occur. In string-motivated supergravity models where supersymmetry is broken via gaugino condensation, there are specific difficulties since, besides a steep dilaton potential preventing the use of this field as the inflaton, it is also difficult to implement the alternative scenario where the dilaton is stabilized and inflation is driven by other fields [四], leading to a runaway problem. Furthermore, at least up to order $\alpha^{\prime 3}$, the structure of the higher order curvature terms does not allow, in the presence of the dilaton, for stable de Sitter type solutions [2]. However, there are some cases where a successful inflationary scenario can be developed as in the so-called dilaton-driven kinetic inflation or Pre-Big-Bang model [3] Jor assuming that the dilaton has been stabilized and that the inflaton is a gauge singlet field other than the dilaton [7, 8, 9] . A successful scenario can also be achieved using hybrid inflationary models [10], where there are two stages of inflation driven by the inflaton and a GUT Higgs field. In Ref. [11], a proposal based on a dual superstring model was made that circumvents the abovementioned difficulties via topological inflation, realizing in a concrete fashion a generic scenario first proposed in [12] to resolve the so-called cosmological moduli problem [13]. In this letter, we analyse other aspects of that model, namely the spectrum of density fluctuations and the reheating temperature.

In Ref. [11] it is shown that, once the requirements of $\mathrm{S}$ and T-duality invariance are imposed, inflation can be achieved via the imaginary part of $\mathrm{S}$ since the conditions for successful inflation are satisfied by domain walls that separate degenerate minima (notice, however, that T-duality alone is not compatible with topological inflation). This model avoids the dilaton runaway problem since the $\mathrm{S}$ and $\mathrm{T}$-dual invariant potential has minima

\footnotetext{
${ }^{1}$ Although this model seems to suffer from a graceful exit problem [n, which cannot be solved with the help of higher-curvature terms [5], there are indications that a quantum cosmological approach may resolve this difficulty [6].
} 
for finite, periodic values of the moduli. Moreover, topological inflation solves the problem of initial conditions for the onset of inflation as, due to the fact that inflation is driven by a topological defect, the field sits necessarily at the top of the potential.

In $N=1$ supergravity, S-duality was conjectured [14] in analogy with T-duality, a wellestablished symmetry of string compactification [15]. Indeed, the target space modular invariance of string effective actions contains a duality transformation as well as discrete shifts of the axionic background. The conjecture is that there would be a further modular invariance symmetry in string theory, where the modular group now acts on the complex scalar field $S=\phi+i \chi$, where $\phi$ is the dilaton and $\chi$ is a pseudoscalar axion field. Notice that this symmetry, which includes S-duality, under which the dilaton gets inverted, strongly constrains the theory since it relates the weak and strong coupling regimes.

Imposing $\mathrm{S}$ and T-duality on the Lagrangian for $N=1$ supergravity theory, one obtains the following potential for a model with 4 moduli, $S, T_{1}, T_{2}$, and $T_{3}$ [ [8] (for models where S-duality is implemented in a different way see [16]):

$$
V_{F}=e^{K}\left|\eta\left(T_{2}\right) \eta\left(T_{3}\right) \eta(S)\right|^{-4}\left(|P|^{2}\left[\frac{S_{R}^{2}}{4 \pi^{2}}\left|\hat{G}_{2}(S)\right|^{2}+\frac{T_{R i}^{2}}{4 \pi^{2}}\left|\hat{G}_{2}\left(T_{i}\right)\right|^{2}-2\right]+F_{0}\right),
$$

where $S_{R}=S+\bar{S}, T_{R i}=T_{i}+\bar{T}_{i}, P=P\left(T_{1}, \psi\right)=\lambda\left(T_{1}\right) \Theta(\psi), \psi$ denotes the untwisted chiral fields related to the $T_{1}$ sector, $\lambda$ and $\Theta$ are gauge invariant functions and $F_{0}=P_{m}\left(K^{-1}\right)_{n}^{m} P^{n}+$ $\left(K_{m} P\left(K^{-1}\right)_{T_{1}}^{m} P^{T_{1}}+\right.$ h.c. $)$, the indices indicate, $m$ and $n$ run over all moduli derivatives and $i=2,3$. The function $\eta(S)=q^{1 / 24} \prod_{n}\left(1-q^{n}\right)$ is the well-known Dedekind function, $q \equiv \exp (-2 \pi S) ; \hat{G}_{2}(S)=G_{2}(S)-2 \pi / S_{R}$ is the weight two Eisenstein function and $G_{2}(S)=$ $\frac{1}{3} \pi^{2}-8 \pi^{2} \sum_{n} \sigma_{1}(n) \exp (-2 \pi n S)$, where $\sigma_{1}(n)$ is the sum of the divisors of $n$, and analogously for the $T_{i}$ moduli. The Kähler function, $K$, and the superpotential, $W$, are given respectively by:

$$
\begin{gathered}
K=-\ln S_{R}-3 \ln T_{R 1}-3 \ln T_{R 2}-3 \ln T_{R 3}, \\
W=\eta(S)^{-2} \eta\left(T_{2}\right)^{-2} \eta\left(T_{3}\right)^{-2} P\left(T_{1}, \psi\right),
\end{gathered}
$$

where the contribution of chiral matter fields was dropped.

Clearly, this potential is $\mathrm{S}$ (and $T_{i}$ )-duality invariant since all dependence on $S$ and $T_{i}$ is given in terms of duality-invariant functions $e^{K}|\eta(S)|^{-4}$ and $S_{R}^{2}\left|\hat{G}_{2}(S)\right|^{2}$. The dual invariant 
points $\left\langle S>=1, e^{-\pi / 6}\right.$ and $<T_{i}>=1, e^{-\pi / 6}$ are extrema (maxima and saddle points, respectively) and the minima of $\mathrm{V}$ are nearby.

In addition to (1), we have to consider the contribution of $D$-terms associated with the gauge sector of the theory, namely

$$
V_{D}=\frac{1}{2 \operatorname{Ref}} D^{2}
$$

where $D=\hat{g} K^{i} T_{i}^{j} \Phi_{j}+\xi, \hat{g}$ being the gauge charge, $T_{i}^{j}$ are the generators of the gauge group and $\xi$ the Fayet-Illiopoulos term. S-duality is ensured for $f=\frac{1}{2 \pi}[\ln (j(S)-744], j(S)$ being the generator of modular invariant functions [16]. From string perturbative results $f=S$ and, therefore, S-duality implies that $f \rightarrow f$.

Assuming that the T-fields and the untwisted fields of the $T_{1}$ sector have already settled at the minimum of the potential so that inflation takes place due to the S-field, the potential of eq. (1) can be then written as

$$
V_{F} \sim\left[\frac{1}{S_{R}|\eta(S)|^{4}}\left(\frac{S_{R}^{2}}{4 \pi^{2}}\left|\hat{G}_{2}(S)\right|^{2}-a\right)\right],
$$

where $a$ parametrises $F_{0}$. Figure 1 shows the potential as a function of Re $\mathrm{S}$ and $\mathrm{Im} \mathrm{S}$, for $a=3$.

In the model of Ref. [6], it is further assumed that $S_{R}$ has settled at the minimum of the potential (at $<S_{R}>\sim 2.6$ ). The total potential, relevant for the computation of density perturbations in our model is, therefore

$$
V=c\left[\frac{1}{<S_{R}>|\eta(S)|^{4}}\left(\frac{<S_{R}^{2}>}{4 \pi^{2}}\left|\hat{G}_{2}(S)\right|^{2}-a\right)+b\right],
$$

where

$$
c \equiv e^{<K>}|P|^{2}<S_{R}>\left|\eta\left(<T_{2}>\right) \eta\left(<T_{3}>\right)\right|^{-4}
$$

and parameter $b$ has been added, representing the contribution of the ground state of $D$ terms, eq. (đ), and ensuring that the potential is positive (this was an implicit assumption in [11]). Notice that, at this stage, S-duality (and also supersymmetry) is broken since a particular non-vanishing vacuum state has been chosen.

\footnotetext{
${ }^{2}$ Another realization of S-duality is $f \rightarrow 1 / f$, but this requires the presence of the so-called "magnetic condensate" [14, 16].
} 
In Ref. [11], it has been shown that the conditions for topological inflation, a scenario first put forward by Linde [10] and Vilenkin [18], to occur at the core of the domain walls separating degenerate minima of the potential can be met for some range of parameters. In this scenario, inflation takes place as the imaginary part of the S field expands exponentially, provided certain conditions are satisfied at the top of the potential. Next, we shall briefly discuss these conditions.

Along a domain wall $\chi$ ranges from one minimum in one region of space to another minimum in another region. Somewhere between, $\chi$ must traverse the top of the potential, $\chi_{0}$, and we hence start expanding the potential about $\chi_{0}$

$$
V \simeq c\left[V_{0}\left(1-\alpha^{2} \frac{\left(\chi-\chi_{0}\right)^{2}}{M^{2}}\right)+b\right],
$$

$M$ being the natural scale of the fields in supergravity and that was set to one in Eqs. (1) - (5). In flat space, the wall thickness is equal to the curvature of the effective potential, that is $\delta^{-1} \simeq \alpha\left(c V_{0} / M_{P}^{2}\right)^{1 / 2}$. The Hubble parameter in the interior of the wall is given by $H \simeq\left(\frac{8}{3} \pi G c \hat{V}_{0}\right)^{1 / 2}$, with $\hat{V}_{0}=V_{0}+b$. If $\delta \ll H^{-1}$, gravitational effects are negligible. However, if $\delta>H^{-1}$, the region of false vacuum near the top of the potential, $V \simeq c \hat{V}_{0}$, extends over a region greater than a Hubble volume. Hence, if the top of the potential satisfies the conditions for inflation, the interior of the wall inflates. Demanding that $\delta>H^{-1}$, one obtains the following condition on $\alpha: \alpha^{2}<\frac{8 \pi}{3}\left(\frac{\hat{V}_{0}}{V_{0}}\right)$. It turns out that this condition is more stringent than the ones that can be derived from demanding an inflationary slow rollover regime [12]. However, the requirement that there are at least $N_{e}$ e-folds of inflation, i.e. $\frac{-V^{\prime \prime}}{V} \ll \frac{6 \pi}{N_{e}}$ leads to the most stringent constraint on $\alpha$ (for $\left.N_{e}=65\right)$ [6]:

$$
\alpha^{2}<\frac{3 \pi}{65}
$$

We have computed $\alpha^{2}=\frac{1}{2} M^{2}\left|\frac{V^{\prime \prime}}{V}\right|$ for different values of $a$ and $b$ and found that, in order to have a positive potential and satisfy the condition (9), we must have $b \geq 8.1 M^{4}$ and

$$
a \geq 2.5
$$

furthermore, we have checked that the slow roll over conditions $|\eta|=M^{2}\left|\frac{V^{\prime \prime}}{V}\right| \leq 1$ and $\epsilon=\frac{1}{2} M^{2}\left(\frac{V^{\prime}}{V}\right)^{2} \leq 1$ are satisfied for any value of $\chi$ in the relevant range, $\chi_{0} \leq \chi \leq \chi_{0}+0.5$. 
Notice that considering the non-canonical struture of the kinetic terms of $S$ (and $T$ ) dictated by $\mathrm{N}=1$ supergravity,$\left(S_{R}\right)^{-2} \partial_{\mu} S \partial^{\mu} S^{*}\left(\left(T_{R}\right)^{-2} \partial_{\mu} T \partial^{\mu} T^{*}\right)$, does not change our results due to modular invariance 3 .

Hence, we conclude that topological inflation is possible for $a \geq 2.5$ and $b \geq 8.1 \mathrm{M}^{4}$, thereby solving the initial condition problems in these models [11].

Of course, in order to have a complete cosmological scenario, it is still required that primordial energy density fluctuations are generated and a successful phase of reheating is achieved. A constraint on the remaining parameter of the superpotential, namely $c$, can be derived from the spectrum of adiabatic density fluctuations, which is given, in terms of the potential, by [20]:

$$
\delta_{H} \equiv \sqrt{4 \pi}\left(\frac{\delta \rho}{\rho}\right)_{H}=\frac{1}{5 \sqrt{3} \pi M^{3}} \frac{V_{N}^{3 / 2}}{V_{N}^{\prime}},
$$

where the subscript $N$ indicates that the right-hand side should be evaluated as the comoving scale $k$ equals the Hubble radius, $k=a(t) H(t)$, during inflation. Neglecting higher multipoles in the Cosmic Microwave Background radiation observed by COBE, the best fit for the quadrupole moment obtained from the angular power spectrum corresponds to [21]

$$
\delta_{H} \approx 2.3 \times 10^{-5}
$$

with an uncertainty of about $10 \%$.

On the other hand, the spectral index $n_{s}$ of the density fluctuations is given in terms of the slow roll over parameters by 20

$$
n_{s} \simeq 1-6 \epsilon\left(\chi_{N}\right)+2 \eta\left(\chi_{N}\right)
$$

In Figure 2, we show $n_{s}(\chi)$ for $a=3$ and $b=8.3 M^{4}$. We see that consistency with observational bounds, i.e. $0.6 \leq n_{s} \leq 1.2$, requires $\chi_{0} \leq \chi_{N} \leq \chi_{0}+0.35$ for the choice $b \gtrsim 8.1 \mathrm{M}^{4}$. Notice that $\delta_{H}$ depends on $c^{1 / 2}$ implying that, in order to satisfy the bound (12), $c$ is constrained to be in the range $1.7 \times 10^{-11} \leq c \leq 4.9 \times 10^{-11}$ (see Figure 3), where we have chosen the values of $\chi$ in the region $\chi_{0}+0.1 \leq \chi \leq \chi_{0}+0.4$ in order to avoid the singularity of Eq. (11) at the extrema of the potential. Hence we can conclude that

\footnotetext{
${ }^{3}$ This point was overlooked in [19], where the periodic structure of the potential in the $\operatorname{ImS}$ direction was approximated by a sinusoidal function, which was then transformed to account for the non-canonical structure of the kinetic terms, a procedure that does not respect modular invariance.
} 


$$
5.0 \times 10^{-10} \leq|P|^{2} \leq 1.4 \times 10^{-9}
$$

as $\eta\left(T_{i}\right)=0.7$ and we have assumed that $<T_{R 1}>\approx<T_{R i}>=2$.

As for the scale dependence of the tensor perturbations we obtain:

$$
n_{t} \simeq-2 \epsilon\left(\chi_{N}\right) \lesssim-5 \times 10^{-4}
$$

meaning that the predicted spectrum of gravitational waves is nearly scale invariant. Furthermore, as the amplitude of the tensor perturbations is given by $\epsilon\left(\chi_{N}\right)^{1 / 2} \delta_{H}$ it follows this is about two orders of magnitude smaller than the amplitude of scalar perturbations.

Once field $\chi$ begins to oscillate about its minimum, the Universe undergoes a reheating phase. At minimum, the inflaton field has a mass $m_{\chi}=\sqrt{2} \gamma$, where $\gamma \lesssim 1.4 \times 10^{-5} \alpha M$. Since the dilaton is hidden from the other sectors of the theory, it couples to lighter fields with strength $\sim \gamma / M$, leading to a decay width

$$
\Gamma_{\chi} \simeq \frac{m_{\chi}}{(2 \pi)^{3}}\left(\frac{\gamma}{M}\right)^{3}
$$

and a reheating temperature

$$
T_{R H}=\left(\frac{30}{\pi^{2} g_{R H}}\right)^{1 / 4} \sqrt{M \Gamma_{\chi}} \simeq \frac{2}{\pi^{2}}\left(\sqrt{\frac{15}{g_{R H}}} \frac{\gamma^{3}}{M}\right)^{1 / 2}
$$

where $g_{R H}$ is the number of degrees of freedom at $T_{R H}$.

A severe upper bound on $T_{R H}$ comes from the requirement that gravitinos are not abundantly regenerated in the post-inflationary reheating epoch. Indeed, once regenerated beyond a certain density, stable thermal gravitinos would dominate the energy density of the Universe or, if they decay, have undesirable effects on nucleosynthesis and light element photo-dissociation and lead to distortions in the microwave background. This implies in bounds of the type 22:

$$
T_{R H} \lesssim(2-6) \times 10^{9} \mathrm{GeV} \quad \text { for } \quad m_{3 / 2}=(1-10) \mathrm{TeV} .
$$

For the model under consideration, for $g_{R H} \approx 150$, we get:

$$
T_{R H} \lesssim 1.4 \times 10^{-9} M=3.4 \times 10^{9} \mathrm{GeV}
$$


However, as discussed in [14], S-duality implies that the gravitino mass is rather high, $O(M)$, in S-dual models without T-duality. In the model of Ref. [8], which is $\mathrm{S}$ and $T_{i^{-}}$ dual, one obtains, after satisfying (12), $m_{3 / 2} \equiv e^{<G>/ 2} M \simeq 1.3 c^{1 / 2} M \lesssim 10^{-5} M$, where $G=K+\ln |W|^{2}$, implying that there is actually no bound on the reheating temperature. In models where one implements S-duality and the possibility of gaugino condensation [16], the gravitino mass can be much smaller and the bounds (18) may turn out to be relevant. Finally we mention that, for our choice of parameters, the vacuum energy density can be estimated as $\rho_{V} \lesssim 2 \times 10^{-10} M^{4}$.

We can then summarize our results as follows. Topological or defect inflation can be achieved in the context of $N=1$ supergravity theories arising from $S$ and $T$ dual string models, as discussed in Ref. [11, and consistency with the observed amplitude of energy density fluctuations can be obtained if the function parametrizing the untwisted fields of the theory satisfies the condition $5.0 \times 10^{-10} \leq|P|^{2} \leq 1.4 \times 10^{-9}, a \geq 2.5$ and $b \geq 8.1 \mathrm{M}^{4}$. This condition ensures that there is no gravitino problem as the gravitino mass is rather high, $m_{3 / 2} \lesssim 10^{-5} \mathrm{M}$. Furthermore, we predict the spectral index to be in the range $0.7 \leq n_{s} \leq 1.2$, as can be seen in Figure 2, and a nearly scale independent spectrum of tensor perturbations, since $n_{t}<<1$. 


\section{References}

[1] R. Brustein and P. J. Steinhardt, Phys. Lett. B302 (1993) 196.

[2] M.C. Bento and O. Bertolami, Phys. Lett. B368 (1996) 198.

[3] M. Gasperini and G. Veneziano, Astropart. Phys. 1 (1993) 317; Phys. Rev. D50 (1994) 2519 ;

J. Levin and K. Freese, Nucl. Phys. B421 (1994) 635.

[4] R. Brustein and G. Veneziano, Phys. Lett. B329 (1994) 429.

[5] N. Kaloper, R. Madden and K.A. Olive, Nucl. Phys. B452 (1995) 677; Phys. Lett. B371 (1996) 34.

[6] M.C. Bento and O. Bertolami, Class. Quantum Gravity, 12 (1995) 1919;

M. Gasperini, J. Maharana and G. Veneziano, Nucl. Phys. B472 (1996) 497;

A. Lukas and R. Poppe, "Decoherence in Pre-Big-Bang Cosmology", hep-th/9603167;

M. P. Dąbrowski and C. Kiefer, Phys. Lett. B397 (1997) 185.

[7] G.G. Ross and S. Sarkar, Nucl. Phys. B461 (1996) 597;

M.C. Bento and O. Bertolami, Phys. Lett. B365 (1996) 59.

[8] A. De La Macorra and S. Lola, Phys. Lett. B373 (1996) 299.

[9] K.-I. Izawa and T. Yanagida, Phys. Lett. B393 (1997) 331.

[10] A.D. Linde, Phys. Lett. B238 (1990) 160; B249 (1990) 18; B259 (1991) 38;

M.C. Bento, O. Bertolami and P.M. Sá, Phys. Lett. B262 (1991) 11; Mod. Phys. Lett. A7 (1992) 911;

E.J. Copeland, A.R. Liddle, D.H. Lyth, E.D. Stewart and D. Wands, Phys. Rev. D49 (1994) 6410;

L. Randall, M. Soljacic and A. Guth, Nucl. Phys. B472 (1996) 377;

G. Dvali, L.M. Krauss and H. Liu, "Hybrid Inflation and Particle Physics", hepph/9707456. 
[11] M.C. Bento and O. Bertolami, Phys. Lett. B384 (1996) 98.

[12] T. Banks, M. Berkooz, S.H. Shenker, G. Moore and P.J. Steinhardt, Phys. Rev. D52 (1995) 3548.

[13] G.D. Coughlan, W. Fischler, E.W. Kolb, S. Raby and G.G. Ross, Phys. Lett. B131 (1983) 59;

J. Ellis, D.V. Nanopoulos and M. Quirós, Phys. Lett. B174 (1986) 176;

O. Bertolami and G.G. Ross, Phys. Lett. B183 (1987) 163;

O. Bertolami, Phys. Lett. B209 (1988) 277;

B. de Carlos, J.A. Casas, F. Quevedo and E. Roulet, Phys. Lett. B318 (1993) 447;

M.C. Bento and O. Bertolami, Phys. Lett. B336 (1994) 6;

T. Banks, D.B. Kaplan and A.E. Nelson, Phys. Rev. D49 (1994) 779;

T. Banks, M. Berkooz and P.J. Steinhardt, Phys. Rev. D52 (1995) 705;

M.C. Bento and O. Bertolami, Gen. Rel. and Gravitation 28 (1996) 565.

[14] A. Font, L.E. Ibáñez, D. Lüst and F. Quevedo, Phys. Lett. B245 (1990) 401; B249 (1990) 35.

[15] E. Alvarez and M. Osorio, Phys. Rev. D40 (1989) 1150.

[16] Z. Lalak, A. Niemeyer and H. P. Nilles, Nucl. Phys. B453 (1995) 100.

[17] A.D. Linde, Phys. Lett. B372 (1994) 208.

[18] A. Vilenkin, Phys. Rev. Lett. 72 (1994) 3137.

[19] D. H. Lyth and E. D. Stewart, Phys. Rev. D54 (1996) 7186;

D. H. Lyth, "Models of inflation and the spectral index of adiabatic density perturbations", hep-ph/9609431.

[20] For reviews: E.W. Kolb and M.S. Turner, The Early Universe (Addison-Wesley, 1990); A.R. Liddle and D.H. Lyth, Phys. Rep. 231 (1993) 1. 
[21] E.L. Wright et al., Astrophys. J. 420 (1994) 1;

K.M. Gorsky et al., Astrophys. J. 430 (1994) L89;

A.J. Banday et al., Astrophys. J. 436 (1994) L99;

C.L. Bennet et al., Astrophys. J. 436 (1994) 423.

[22] J. Ellis, G.B. Gelmini and S. Sarkar, Nucl. Phys. B373 (1992) 399;

S. Sarkar, Rep. Prog. Phys. 59 (1996) 1493. 


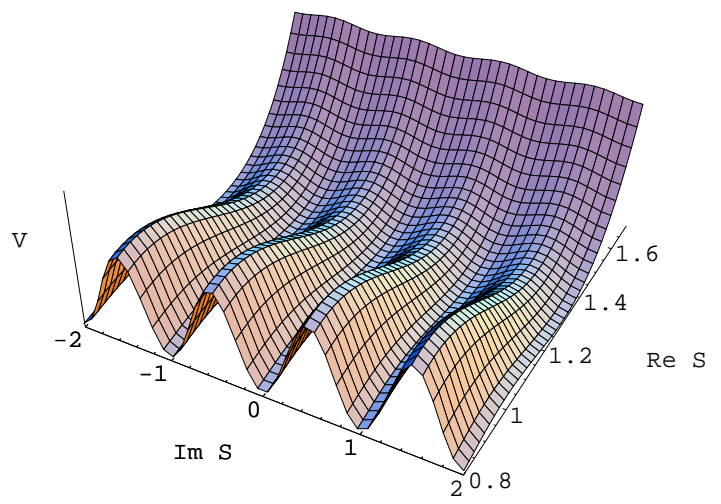

Figure 1: The potential as a function of Re $\mathrm{S}$ and $\operatorname{Im} \mathrm{S}$ (for $a=3, b=8.3 M^{4}$ and $c=1$, see Eq. (4)).

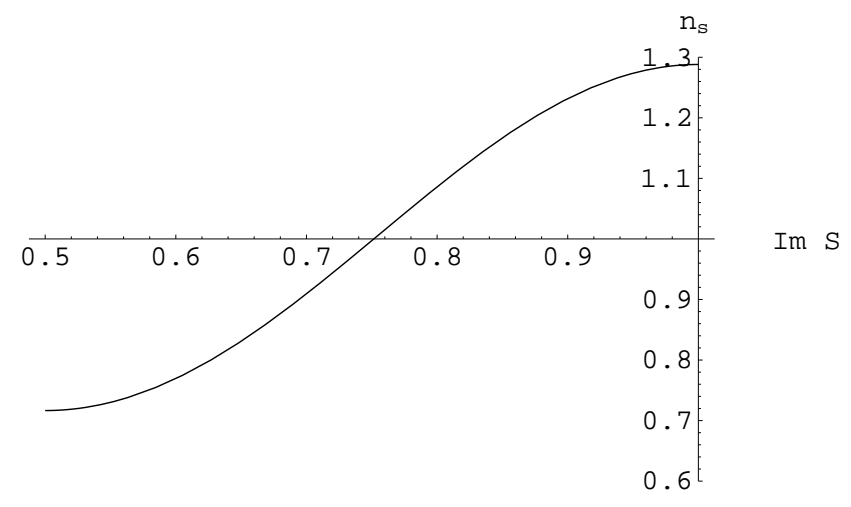

Figure 2: The spectral index $n_{s}$ as a function of $\chi\left(a=3\right.$ and $\left.b=8.3 M^{4}\right)$. 


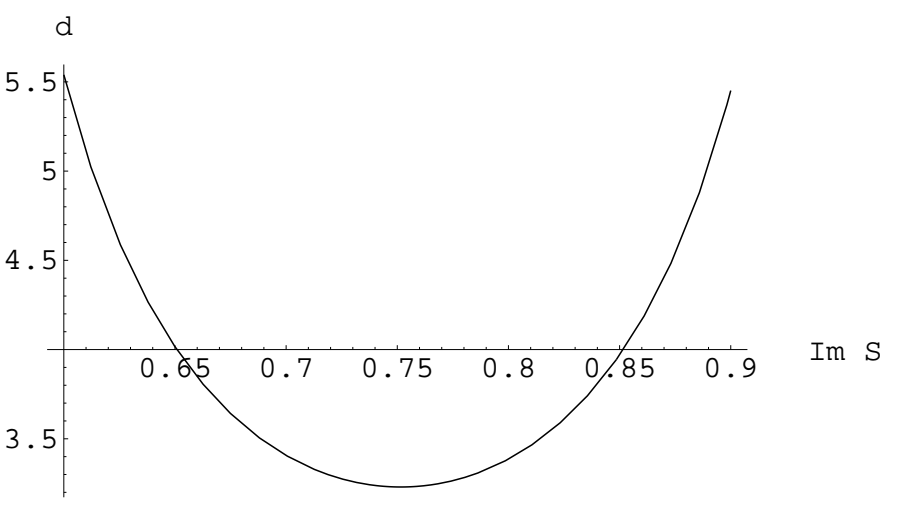

Figure 3: The density fluctuation parameter $d \equiv \delta_{H} / c^{1 / 2}$ as a function of $\chi(a=3$ and $\left.b=8.3 M^{4}\right)$. 